Ophthalmologists are well aware of the ocular risks of EL and routinely survey for these. However, ensuring cardiovascular assessments may be less-regularly documented. Herein we audit documentation of whether patients with EL are known to cardiologists, geneticists or physicians for their systemic cardiovascular risks.

Eligible patients were identified from the database of a tertiary ophthalmic centre: Moorfields Eye Hospital, London. A database search between 1 January 1959 and 31 December 2011 was performed. The inclusion criteria were all referrals for EL or for syndromes associated with EL. Patients were excluded if the EL diagnosis was not confirmed, or if patients were subsequently seen elsewhere.

The standard of the audit was that all patients should have documentation that the patient is under cardiovascular review.

In all, 191 patients fulfilled the inclusion criteria. Ninetyseven patients $(51 \%)$ had documentation that they were under cardiovascular review. Of these, 38 (20\%) were documented to be known as having cardiovascular issues, while 59 patients (31\%) did not. A total of 94 patients (49\%) had no documentation regarding cardiovascular problems.

Our findings describe the lack of documentation ensuring cardiovascular review for patients with EL. Ophthalmologists are well aware that the most common cause of EL is MFS and associated cardiovascular risks. It is important for patient safety that these patients are known to cardiology or medical services. Ophthalmologists first see $\sim 40 \%$ of patients with MFS [7]. It is critical therefore to ensure that patients with EL are under the care of appropriate physicians. There are currently no guidelines on referral pathways for patients who present to ophthalmologists with EL, suggesting the need to review investigation and management of this cohort.

\section{Compliance with ethical standards}

Conflict of interest The authors declare that they have no conflict of interest.

\section{References}

1. Stone JH. Ectopia lentis, cardiology, and the sign of the tremulous iris. Am Heart J. 1966;72:466-8.

2. Chandra A, Charteris D. Molecular pathogenesis and management strategies of ectopia lentis. Eye 2014;28:162-8.

3. Marsalese DL, Moodie DS, Vacante M, Lytle BW, Gill CC, Sterba $\mathrm{R}$, et al. Marfan's syndrome: natural history and long-term followup of cardiovascular involvement. J Am Coll Cardiol. 1989;14: 422-8-31.

4. Rubin S, Nelson L. Ocular manifestations of autosomal dominant systemic conditions. In: Tasman W, Jaegar E, editors. Duane's clinical ophthalmology. 2nd ed. Lippincott Williams and Wilkins; Philadelphia 2006.

5. Graham Stuart A, Williams A. Marfan's syndrome and the heart. Arch Dis Child. 2007;92:351-6.

6. Child AH. Diagnosis and management (Summary). In: Diagnosis and management of Marfan Syndrome. (ed.) Child, Anne H. London: Springer; 2016. p. 3-11.

7. Strider D, Moore T, Guarini J, Fallin B, Ivey J, Kron I. Marfan's syndrome: a family affair. J Vasc Nurs. 1996;14:91-98.

\title{
Periocular basal cell carcinoma in under 40's - Is more aggressive treatment warranted?
}

\author{
Edward Saxby $^{1} \cdot$ Mark Wright $^{1} \cdot$ Rhys Davies $^{1}$
}

Received: 15 November 2018 / Accepted: 15 November 2018 / Published online: 5 December 2018

(c) The Royal College of Ophthalmologists 2018

Edward Saxby

e_saxby@hotmail.com

1 Princess Alexandra Eye Pavilion, Chalmers Street, Edinburgh EH3 9HA NHS Lothian, UK
Basal cell carcinoma (BCC) is the most common form of skin cancer. It is slow growing and locally invasive. Risk factors include ultraviolet light exposure, increasing age, fair skin, immunosuppresion and familial conditions e.g. Gorlins Syndrome [1]. BCCs are commonly diagnosed in elderly populations but are still rarely diagnosed in youth [2]. We investigated the incidence, treatment and outcome 
Table 1 Characteristics of BCCs in the under 40's

\begin{tabular}{ll}
\hline Age (mean) & 33.8 years ${ }^{(+/-4.1 \mathrm{SD})}$ \\
\hline Sex & 6 Males, 7 Females \\
Pathology & 8 Nodular, 4 Morphoeic, 1 \\
& $\begin{array}{l}\text { Basisquamous } \\
\text { Time from onset of signs to }\end{array}$ \\
treatment (mean) & \\
Recurrence & 3 patients \\
Time to recurrence (mean) & 2.3 years ${ }^{(+/-0.6 \mathrm{SD})}$ \\
\end{tabular}

of patients under 40 years of age with a histologically confirmed diagnosis of a periocular BCC.

A retrospective analysis of all patients that attended a 'tumour clinic' at the Edinburgh Princess Alexandra Eye Pavilion, over a 17-year period, was performed. Cases were identified from pathology diaries in which all patients that undergo a biopsy for suspected periocular malignancy are logged. This allowed us to identify all patients under the age of 40 that had a histologically confirmed BCC. ${ }^{1}$

Over the 17-year period 1068 biopsies were performed. Pathology results confirmed 720 of the biopsies were BCCs. We found 13 BCCs present in the under 40's population with a mean length of follow up of 31 months ${ }^{(+/-21 \mathrm{SD})}$ (Table 1).

We have shown a prevalence of periocular BCC's in our under 40's population of $1.8 \%$ which is in keeping with national data showing a BCC prevalence of $1.2 \%$ in patients aged 15-35 years [3].

Recurrence was defined as histologically confirmed $\mathrm{BCC}$, arising at the site of previous surgery. A recurrence rate of $23.1 \%$ in our 'under 40 's' population is 12 times greater than our overall audited recurrence rate of $1.9 \%$, with 17 year follow up, and the national average of $<2 \%$, with a five year follow up [2]. None of the recurrence cases had any underlying risk factors.
Margins of $2 \mathrm{~mm}$ are our standard practice. In one case of recurrent tumour, to preserve the lid margin, the surgical excision was less than $2 \mathrm{~mm}$ superiorly. Two of the three cases of recurrent tumour were noted to be morphoeic and in both cases the tumours were completely excised according to histology. Recurrence following histologically confirmed complete excision occurs in $1.3 \%$ of cases [3]. Our findings highlight the need for ongoing follow up irrespective of the histology report.

The time from onset of signs/symptoms to treatment was 2.7 years $^{(+/-1.1 \mathrm{SD})}$ in our study population. We believe this demonstrates a significant diagnostic delay.

BCCs in under 40's are rare; however the recurrence rate in this population is 12 times higher than our overall audited recurrence rate. This may reflect a higher proportion of morphoeic tumours, associated with an increased recurrence rate, or an intrinsically more aggressive disease. Given the significantly higher recurrence rate for patients under 40 , we would recommend larger excision margins or excision with Moh's micrographic technique and a prolonged follow up period.

\section{Compliance with ethical standards}

Conflict of interest The authors declare that they have no conflict of interest.

\section{References}

1. Foundation TBS. Basal Cell Carcinoma http://www. britishskinfoundation.org.uk/SkinInformation/AtoZofSkindisease/ BasalCellCarcinoma.aspx.

2. Pandeya NOC, Whiteman D. The incidence and multiplicity rates of keratinocyte cancers in Australia. Med J Aust. 2017;207:339-43.

3. Basal NC. Cell Carcinoma in young Adults. Br J Dermatol. 1992;127:26-9.

\footnotetext{
1 This project was orally presented at the British Oculoplastics Society Meeting in Dublin June 2018.
} 